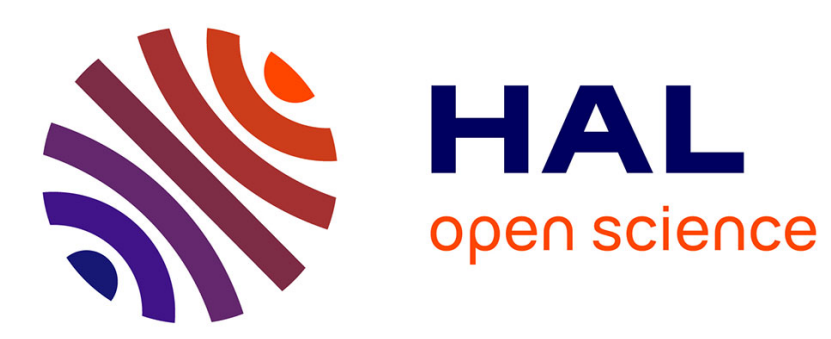

\title{
Isofulminic acid, HONC: Ab initio theory and microwave spectroscopy
}

M. Mladenovic, Marius Lewerenz, M.C. Mccarthy, P. Thaddeus

\section{To cite this version:}

M. Mladenovic, Marius Lewerenz, M.C. Mccarthy, P. Thaddeus. Isofulminic acid, HONC: Ab initio theory and microwave spectroscopy. Journal of Chemical Physics, 2009, 131 (17), pp.174308. 10.1063/1.3257680 . hal-00750631

\section{HAL Id: hal-00750631 \\ https://hal.science/hal-00750631}

Submitted on 12 Nov 2012

HAL is a multi-disciplinary open access archive for the deposit and dissemination of scientific research documents, whether they are published or not. The documents may come from teaching and research institutions in France or abroad, or from public or private research centers.
L'archive ouverte pluridisciplinaire HAL, est destinée au dépôt et à la diffusion de documents scientifiques de niveau recherche, publiés ou non, émanant des établissements d'enseignement et de recherche français ou étrangers, des laboratoires publics ou privés. 


\section{AIP Chemicital Prysics}

\section{Isofulminic acid, HONC: Ab initio theory and microwave spectroscopy}

Mirjana Mladenović, Marius Lewerenz, Michael C. McCarthy, and Patrick Thaddeus

Citation: J. Chem. Phys. 131, 174308 (2009); doi: 10.1063/1.3257680

View online: http://dx.doi.org/10.1063/1.3257680

View Table of Contents: http://jcp.aip.org/resource/1/JCPSA6/v131/i17

Published by the American Institute of Physics.

\section{Additional information on J. Chem. Phys.}

Journal Homepage: http://jcp.aip.org/

Journal Information: http://jcp.aip.org/about/about_the_journal

Top downloads: http://jcp.aip.org/features/most_downloaded

Information for Authors: http://jcp.aip.org/authors

\section{ADVERTISEMENT}

\section{ACCELERATE COMPUTATIONAL CHEMISTRY BY 5X. TRY IT ON A FREE, REMOTELY-HOSTED CLUSTER. \\ nVIDIA.}




\title{
Isofulminic acid, HONC: $\boldsymbol{A b}$ initio theory and microwave spectroscopy
}

\author{
Mirjana Mladenović, ${ }^{1, a)}$ Marius Lewerenz, ${ }^{1, b)}$ Michael C. McCarthy, ${ }^{2, c)}$ and \\ Patrick Thaddeus ${ }^{2, d}$ \\ ${ }^{1}$ Laboratoire Modélisation et Simulation Multi Echelle, MSME FRE3160 CNRS, Université Paris-Est, \\ 5 bd Descartes, 77454 Marne la Vallée, Cedex 2, France \\ ${ }^{2}$ Harvard-Smithsonian Center for Astrophysics, 60 Garden Street, Cambridge, Massachusetts 02138, USA \\ and School of Engineering and Applied Sciences, Harvard University, Cambridge, \\ Massachusetts 02138, USA
}

(Received 25 June 2009; accepted 9 October 2009; published online 4 November 2009)

\begin{abstract}
Isofulminic acid, $\mathrm{HONC}$, the most energetic stable isomer of isocyanic acid $\mathrm{HNCO}$, higher in energy by $84 \mathrm{kcal} / \mathrm{mol}$, has been detected spectroscopically by rotational spectroscopy supported by coupled cluster electronic structure calculations. The fundamental rotational transitions of the normal, carbon-13, oxygen-18, and deuterium isotopic species have been detected in the centimeter band in a molecular beam by Fourier transform microwave spectroscopy, and rotational constants and nitrogen and deuterium quadrupole coupling constants have been derived. The measured constants agree well with those predicted by ab initio calculations. A number of other electronic and spectroscopic parameters of isofulminic acid, including the dipole moment, vibrational frequencies, infrared intensities, and centrifugal distortion constants have been calculated at a high level of theory. Isofulminic acid is a good candidate for astronomical detection with radio telescopes because it is highly polar and its more stable isomers (HNCO, HOCN, and HCNO) have all been identified in space. (C) 2009 American Institute of Physics. [doi:10.1063/1.3257680]
\end{abstract}

\section{INTRODUCTION}

Isocyanic acid and its isomers have long attracted organic chemists because they are composed of the four most important biogenic elements of organic chemistry, and because they provide interesting tests of chemical bonding and electronic structure. The isocyanic acid isomers have been the subject of theoretical and experimental studies since the 16th century. ${ }^{1}$ Although many isomeric arrangements are possible, only four are minima on the potential energy surface: $^{2-4}$ isocyanic acid HNCO, cyanic acid HOCN, fulminic acid HCNO, and isofulminic acid HONC. All have singlet electronic ground states, linear or nearly linear heavyatom backbones, and planar equilibrium structures. ${ }^{3-5}$ Recent calculations ${ }^{5}$ predict that HOCN, HCNO, and HONC lie, respectively, 24.7, 70.7, and $84.1 \mathrm{kcal} / \mathrm{mol}$ higher in energy than HNCO. All are calculated to be highly polar molecules, with dipole moments from 1 to $3.5 \mathrm{D}^{6,7}$

The isocyanic isomers are of astronomical interest. Isocyanic acid itself was one of the first molecules ${ }^{8}$ identified in the interstellar gas on the basis of the prior laboratory microwave spectroscopy. ${ }^{9}$ It has now been found in many locations, including over sixty galactic sources and nine external galaxies. ${ }^{10-14}$ On the basis of recent laboratory data, there is now evidence for cyanic acid in the Galactic center source Sgr B2 ${ }^{15,16}$ at about the same time, Marcelino et al. ${ }^{17}$ observed radio lines of the third most stable isomer, fulminic

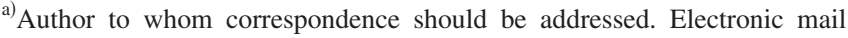
mladenov@univ-mlv.fr.

${ }^{b)}$ Electronic mail: lewerenz@univ-mlv.fr.

${ }^{c}$ Electronic mail: mccarthy@cfa.harvard.edu.

${ }^{\mathrm{d})}$ Electronic mail: pthaddeus@cfa.harvard.edu.
}

acid, in three starless cores and in the low-mass star forming region L1527. The presence of higher-lying isomers in hot cores and other warm regions may result from reactions on the surfaces of interstellar dust grains. If so, the isocyanic isomers may provide a useful tool to estimate relative contributions of gas-phase and grain chemistry.

Until quite recently, only isocyanic acid and fulminic acid had been detected at high spectral resolution in the gas phase. The rotational and vibrational spectra of both have now been thoroughly studied, ${ }^{18,19}$ motivated partly by a desire to understand the quasilinear structure produced by the large-amplitude bending motion. The second most stable isomer, cyanic acid HOCN, was first detected at low temperature in an inert matrix after ultraviolet irradiation of $\mathrm{HNCO}^{20}$ work extended by Bondybey et al. ${ }^{21}$ who showed that irradiation of HCNO in a matrix also yields HOCN. During the past year, cyanic acid has been detected in the gas phase, and its microwave rotational spectrum measured. ${ }^{15}$

Until the present work, there was no conclusive evidence for the remaining isomer, isofulminic acid HONC. Maier et $a l^{22}$ reported a low temperature infrared matrix spectrum in argon, but the assignment was subsequently withdrawn. ${ }^{23}$ There have been at least two attempts to produce HONC by pyrolysis of mercury fulminate and other compounds; the first by Wentrup et al. ${ }^{24}$ only produced HCNO and trace amounts of $\mathrm{HNCO}$; the second by McNaughton and Evans ${ }^{25}$ yielded a weak feature near the predicted frequency of the $3_{0,3} \rightarrow 2_{0,2}$ rotational transition of HONC, without confirming lines. No lines attributable to HOCN were observed.

From theoretical self-consistent field (SCF) calculations of the potential energy surface of the isocyanic isomers, Poppinger $e t a l .^{3}$ and McLean $e t a l^{2}$ concluded that isofulminic 
acid is stable enough to exist in the interstellar gas. Poppinger et $\mathrm{al}^{26}$ also examined substituted isocyanic acid isomers, in particular the stability of the lesser known RONC isomers, first experimentally observed by Wentrup et al. ${ }^{27}$ three years later. Pinnavaia $e t a .^{4}$ used a perturbational approach to calculate spectroscopic parameters of HONC from a quartic internal coordinate force field determined by second order Møller-Plesset theory. Isomerization and fragmentation pathways for the CHNO system were studied by Mebel et al. ${ }^{28}$ with density functional theory, by Ding et al. ${ }^{29}$ with the complete active space method, and by Schuurman $e t$ $a l .{ }^{5}$ with several coupled cluster approaches. The equilibrium geometry and energetics of the four most stable isocyanic isomers were recently analyzed in significant detail by some of the present authors with the all electron coupled cluster singles and doubles with perturbative triple excitations, $\operatorname{CCSD}(\mathrm{T})$ method, in combination with large correlation consistent bases. ${ }^{6,30}$ The computed barriers to linearity from all electron CCSD(T)/aug-cc-pCVQZ calculations are $5.3 \mathrm{kcal} /$ $\mathrm{mol}\left(1839 \mathrm{~cm}^{-1}\right)$ for HNCO, $26.9 \mathrm{kcal} / \mathrm{mol}\left(9406 \mathrm{~cm}^{-1}\right)$ for HOCN, and $47.9 \mathrm{kcal} / \mathrm{mol}\left(16761 \mathrm{~cm}^{-1}\right)$ for HONC, whereas the equilibrium structure of $\mathrm{HCNO}$ was calculated to be linear. In addition, HOCN, HCNO, and HONC were found to lie $24.5 \mathrm{kcal} / \mathrm{mol}\left(8577 \mathrm{~cm}^{-1}\right), 70.0 \mathrm{kcal} / \mathrm{mol}$ $\left(24467 \mathrm{~cm}^{-1}\right)$, and $84.2 \mathrm{kcal} / \mathrm{mol}\left(29464 \mathrm{~cm}^{-1}\right)$ above the most stable HNCO molecule, in good agreement (to $0.2 \mathrm{kcal} /$ $\mathrm{mol}$ ) with the earlier frozen-core focal-point analysis results of Schuurman et al. ${ }^{5}$ Minimum energy paths along the angular coordinates were used in combination with the dominant kinetic energy contributions to predict their key rovibrational spectroscopic features. ${ }^{30}$ The harmonic heavy-atom bending frequency $\omega_{5}$ and the harmonic torsional frequency $\omega_{6}$ of HONC were identified as the lowest among all of the four stable CHNO isomers. ${ }^{6,30}$ Their electric and magnetic properties were studied in Ref. 7, where both a Mulliken population analysis of the total electron density and molecular orbitals were used to interpret the changes in the electronic structure properties.

Motivated by the recent gas-phase detection of cyanic acid $^{15}$ and coupled cluster calculations, ${ }^{6,7,30}$ a combined experimental and theoretical effort has been undertaken to spectroscopically characterize the most energetic isomer, isofulminic acid. Here we report the laboratory detection of the fundamental rotational transition of HONC in a supersonic molecular beam by Fourier transform microwave (FTM) spectroscopy. Our identification has been confirmed by detection of the same transition in the carbon-13, oxygen18 , and deuterium isotopic species, with the expected nitrogen hyperfine structure. A number of other spectroscopic quantities including equilibrium and ground state rotational constants, dipole moments, anharmonic vibrational frequencies, infrared intensities, and centrifugal distortion constants have also been calculated theoretically for HONC and its rare isotopologues.

\section{THEORETICAL CALCULATIONS}

$A b$ initio calculations have been carried out for the ground $A^{\prime}$ electronic state by means of the coupled cluster

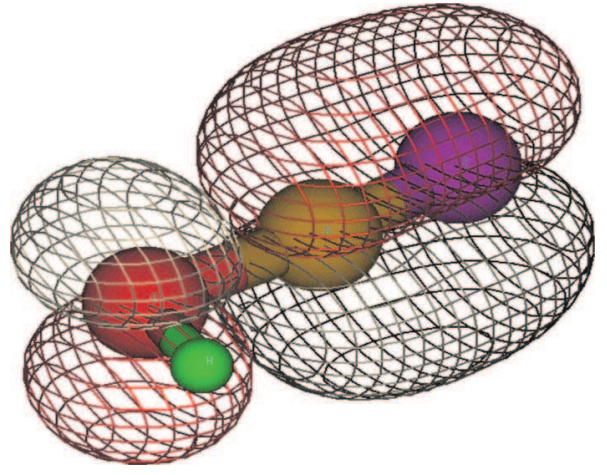

FIG. 1. Highest occupied molecular $2 a^{\prime \prime}$ orbital of HONC. The contour surfaces of orbital amplitude 0.025 and -0.025 are shown.

electron correlation method $\operatorname{CCSD}(\mathrm{T})$ including all single and double excitations and a perturbative term for connected triple substitutions, as implemented in the Mainz-AustinBudapest version of the ACES2 program package. ${ }^{31}$ Dunning's correlation consistent polarized core valence basis sets, commonly termed cc-pCVXZ, have been used. ${ }^{32,33}$

HONC possesses a planar equilibrium structure of trans type. ${ }^{7}$ In $C_{s}$ symmetry, the reference electronic configuration used in the SCF approximation to construct molecular orbitals is given by

$$
(\text { core })^{6}\left(4 a^{\prime}\right)^{2}\left(5 a^{\prime}\right)^{2}\left(6 a^{\prime}\right)^{2}\left(7 a^{\prime}\right)^{2}\left(1 a^{\prime \prime}\right)^{2}\left(8 a^{\prime}\right)^{2}\left(9 a^{\prime}\right)^{2}\left(2 a^{\prime \prime}\right)^{2},
$$

where the core contribution $\left(1 a^{\prime}\right)^{2}\left(2 a^{\prime}\right)^{2}\left(3 a^{\prime}\right)^{2}$ contains the three $1 s$-like core orbitals located on oxygen, nitrogen, and carbon, respectively. The highest occupied molecular $2 a^{\prime \prime}$ orbital is primarily composed of the out-of-plane $2 p_{x}$ atomic orbitals of $\mathrm{O}, \mathrm{C}$, and $\mathrm{N}$, as depicted in Fig. 1, where a lone electron pair on oxygen may easily be noted. The dominant single determinant reference character is confirmed by a $T_{1}$ diagnostic value near 0.014 and largest $\left|T_{2}\right|$ amplitudes smaller than 0.07 in our all electron $\operatorname{CCSD}(\mathrm{T})$ calculations.

\section{A. Equilibrium geometry}

Large-scale coupled cluster $\operatorname{CCSD}(\mathrm{T})$ all electron results for the equilibrium structure of HONC were reported in a recent study on the CHNO isomer family. ${ }^{30}$ There the geometrical parameters at the complete basis set (CBS) limit, computed for the cc-pCVXZ and aug-cc-pCVXZ basis set families by means of the simple three-parameter exponential extrapolation, showed excellent agreement within $0.0003 \mathrm{a}_{0}$, $0.02^{\circ}[\angle(\mathrm{ONC})]$, and $0.07^{\circ}[\angle(\mathrm{HON})]$. The best estimate for the heavy atom $\mathrm{ONC}$ bond angle of $172.56^{\circ}$, introduced in Ref. 30 as the mean value of the two respective CBS results, is employed in the present work in combination with the $\operatorname{CCSD}(\mathrm{T}) / \mathrm{cc}-\mathrm{pCV} \infty \mathrm{Z}$ bond lengths and the mean value of $104.92^{\circ}$ for the HON angle, where $\infty$ denotes the CBS limit. The latter set of geometrical parameters is displayed in Fig. 2 and will be referred to as the best estimate geometry. For quantities computed at this geometrical arrangement, we use the superscript $\infty$ throughout this paper.

The NC bond length in Fig. 2 is only $0.004 \AA$ longer than $r(\mathrm{NC})$ of $1.169 \AA$ in $\mathrm{HNC},{ }^{34}$ indicating a similar type of bonding $\left(\mathrm{N}^{+} \equiv \mathrm{C}^{-}\right)$in the two molecules. This is in accord with a partial atomic charge $(\delta)$ distribution found to be 


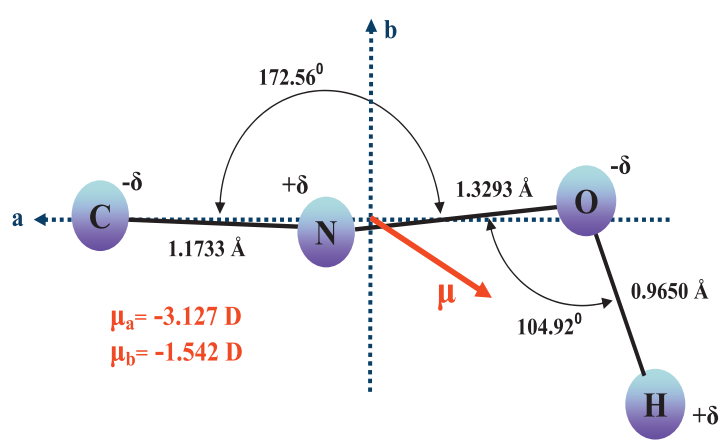

FIG. 2. A $b$ initio equilibrium structure, principal $a, b$ moment-of-inertia axes and dipole moment vector $\mu$.

$+0.31,-0.24,+0.14$, and $-0.21 e$ along the $\mathrm{H}-\mathrm{O}-\mathrm{N}-\mathrm{C}$ chain from the atomic population analysis of the $\operatorname{CCSD}(\mathrm{T}) / \mathrm{cc}-$ pCV5Z density (see Sec. II D). One may also note that the $\mathrm{H}-\mathrm{O}-\mathrm{N}$ angle in Fig. 2 compares well with the $\mathrm{H}-\mathrm{O}-\mathrm{H}$ angle of $104.45^{\circ}$ in $\mathrm{H}_{2} \mathrm{O},{ }^{35}$ what presumably points at a similar spatial electron distribution around oxygen in HONC and $\mathrm{H}_{2} \mathrm{O}$.

The $a$ principal inertial axis in Fig. 2 lies rather close to the nearly linear heavy-atom ONC backbone of the molecule, rotated only $4.8^{\circ}$ from the $\mathrm{N}-\mathrm{O}$ bond. The $\operatorname{CCSD}(\mathrm{T}) /$ cc-pCV5Z dipole moment vector for the best estimate structure has a magnitude of $|\mu|=3.487 \mathrm{D}$. This value is somewhat smaller than $|\mu|=3.815 \mathrm{D}$ for HOCN and larger than $|\mu|=3.340$ for HCNO and $|\mu|=2.073$ D for HNCO, calculated at the same $\operatorname{CCSD}(\mathrm{T}) /$ cc-pCV5Z level of theory. ${ }^{7}$ In addition, $|\mu|$ of HONC is somewhat larger than the $\operatorname{CCSD}(\mathrm{T}) / \mathrm{aug}$-cc-pCVQZ dipole moment of 3.110 D calcu- lated here for $\mathrm{HNC}$. The experimentally derived $|\mu|$ values for HNCO, HCNO, and HNC are 2.075 D (Ref. 36), 3.350 D (Ref. 37), and 3.050 D (Ref. 38), respectively.

\section{B. Spectroscopic parameters}

Zero point vibrational corrections to rotational constants could be computed only at the $\operatorname{CCSD}(\mathrm{T}) / \mathrm{cc}-\mathrm{pCVTZ}$ level due to technical limitations. The equilibrium rotational constants calculated from the $\operatorname{CCSD}(\mathrm{T}) / \mathrm{cc}$-pCVTZ optimum geometry reported previously ${ }^{30}$ and from the best estimate structure of Fig. 2 are collected for the five HONC isotopologues in Table I. The former set of constants is denoted by $A_{e}^{(3)}, B_{e}^{(3)}, C_{e}^{(3)}$, whereas we use $A_{e}^{(\infty)}, B_{e}^{(\infty)}, C_{e}^{(\infty)}$ to designate the latter results. As seen, HONC is an almost prolate symmetric top with an asymmetry parameter $\kappa$ of -0.9994 . The ground-state rotational constants $A_{0}^{(3)}, B_{0}^{(3)}, C_{0}^{(3)}$ and groundstate vibrational corrections $\Delta A^{(3)}, \Delta B^{(3)}, \Delta C^{(3)}$ are both obtained from the $\operatorname{CCSD}(\mathrm{T}) / \mathrm{cc}-\mathrm{pCVTZ}$ calculations carried out at the $\operatorname{CCSD}(\mathrm{T}) / \mathrm{cc}-\mathrm{pCVTZ}$ optimum geometry. The groundstate vibrational corrections are computed from the cubic and semidiagonal quartic force field in a normal coordinate representation by means of vibrational second-order perturbation theory. ${ }^{39}$

The ground-state rotational constants for the best $\operatorname{CCSD}(\mathrm{T})$ estimate structure are obtained from $A_{e}^{(\infty)}, B_{e}^{(\infty)}, C_{e}^{(\infty)}$ and the ground-state $\mathrm{CCSD}(\mathrm{T}) / \mathrm{cc}$-pCVTZ vibrational corrections according to

TABLE I. Experimental and theoretical rotational constants. Equilibrium rotational constants $A_{e}, B_{e}, C_{e}$, ground-state rotational constants $A_{0}, B_{0}, C_{0}$, and ground-state vibrational corrections $\Delta A, \Delta B, \Delta C$ are calculated from the $\operatorname{CCSD}(\mathrm{T}) / \mathrm{cc}$-pCVTZ optimum structure (superscript 3) and the best $\operatorname{CCSD}(\mathrm{T})$ estimate structure (superscript $\infty$ ). The experimentally derived rotational constants $B_{\text {eff }}$ for the $K_{a}=0$ ladder are highlighted by bold numbers. The numbers in parentheses given along with the experimental values are the estimated uncertainties $(1 \sigma)$ in units of the last significant digit. For the definition of $\bar{B}_{\text {eff }}^{(\infty, 3)}$ and $\Delta\left(B_{\text {eff }}\right)$, see Eqs. (2) and (3). All quantities are given in megahertz.

\begin{tabular}{lcccrr}
\hline \hline Property & HONC & DONC & HON ${ }^{13} \mathrm{C}$ & $\mathrm{HO}^{15} \mathrm{NC}$ & $\mathrm{H}^{18} \mathrm{ONC}$ \\
\hline$A_{e}^{(3)}$ & 616751 & 341630 & 616606 & 615722 & 609174 \\
$\Delta A^{(3)}$ & -1888 & 1222 & -1981 & -2326 & -2486 \\
$A_{0}^{(3)}$ & 618638 & 340407 & 618586 & 618048 & 611660 \\
$B_{e}^{(3)}$ & 11025 & 10547 & 10592 & 11015 & 10471 \\
$\Delta B^{(3)}$ & 36 & 27 & 34 & 36 & 34 \\
$B_{0}^{(3)}$ & 10989 & 10519 & 10558 & 10979 & 10437 \\
$C_{e}^{(3)}$ & 10831 & 10231 & 10413 & 10821 & 10294 \\
$\Delta C^{(3)}$ & 51 & 46 & 47 & 51 & 47 \\
$C_{0}^{(3)}$ & 10781 & 10185 & 10366 & 10770 & 10247 \\
$A_{e}^{(\infty)}$ & 622335 & 345246 & 622186 & 621255 & 614597 \\
$B_{e}^{(\infty)}$ & 11107 & 10619 & 10671 & 11097 & 10549 \\
$C_{e}^{(\infty)}$ & 10912 & 10302 & 10491 & 10902 & 10371 \\
$A_{0}^{(\infty, 3)}$ & 624223 & 344024 & 624167 & 623581 & 617083 \\
$B_{0}^{(\infty, 3)}$ & 11071 & 10592 & 10637 & 11061 & 10515 \\
$C_{0}^{(\infty, 3)}$ & 10861 & 10256 & 10444 & 10851 & 10330 \\
$\bar{B}_{\text {eff }}^{(\infty, 3)}$ & 10966 & 10424 & 10540 & 10956 & 10422 \\
$\boldsymbol{B}_{\text {eff }}$ & $\mathbf{1 0 9 5 3 . 3 9 0 4 ( 4 )}$ & $\mathbf{1 0 4 1 1 . 8 2 3 8 ( 6 )}$ & $\mathbf{1 0 5 2 8 . 2 9 8 5 ( 6 )}$ & & $\mathbf{1 0 4 0 7 . 9 8 7 0 4 ( 6 )}$ \\
$\Delta\left(B_{\text {eff }}\right)$ & 13 & 12 & 12 & & 14 \\
\hline \hline
\end{tabular}


TABLE II. CCSD(T)/cc-pCVTZ harmonic $\omega_{i}$ and fundamental $\nu_{i}$ vibrational frequencies (in $\mathrm{cm}^{-1}$ ), as well as the infrared intensity $I$ (in $\mathrm{km} / \mathrm{mol}$ ), computed for the CCSD(T)/cc-pCVTZ optimum geometry.

\begin{tabular}{lccccc}
\hline \hline Property & HONC & DONC & HON ${ }^{13} \mathrm{C}$ & $\mathrm{HO}^{15} \mathrm{NC}$ & $\mathrm{H}^{18} \mathrm{ONC}$ \\
\hline$\omega_{1}\left(a^{\prime}\right)$ & 3770 & 2746 & 3770 & 3770 & 3758 \\
$\omega_{2}\left(a^{\prime}\right)$ & 2170 & 2167 & 2137 & 2129 & 2168 \\
$\omega_{3}\left(a^{\prime}\right)$ & 1435 & 1070 & 1434 & 1433 & 1430 \\
$\omega_{4}\left(a^{\prime}\right)$ & 968 & 966 & 957 & 964 & 936 \\
$\omega_{5}\left(a^{\prime}\right)$ & 252 & 245 & 250 & 246 & 251 \\
$\omega_{6}\left(a^{\prime \prime}\right)$ & 305 & 300 & 302 & 298 & 303 \\
$\nu_{1}\left(a^{\prime}\right)$ & 3584 & 2648 & 3584 & 3584 & 3573 \\
$\nu_{2}\left(a^{\prime}\right)$ & 2128 & 2125 & 2095 & 2089 & 2126 \\
$\nu_{3}\left(a^{\prime}\right)$ & 1393 & 1047 & 1392 & 1392 & 1389 \\
$\nu_{4}\left(a^{\prime}\right)$ & 949 & 947 & 938 & 945 & 917 \\
$\nu_{5}\left(a^{\prime}\right)$ & 273 & 257 & 270 & 267 & 271 \\
$\nu_{6}\left(a^{\prime \prime}\right)$ & 321 & 308 & 318 & 314 & 319 \\
$I\left(\nu_{1}\right)$ & 108 & 54 & 108 & 108 & 108 \\
$I\left(\nu_{2}\right)$ & 20 & 17 & 18 & 20 & 21 \\
$I\left(\nu_{3}\right)$ & 60 & 38 & 61 & 60 & 58 \\
$I\left(\nu_{4}\right)$ & 41 & 35 & 40 & 41 & 39 \\
$I\left(\nu_{5}\right)$ & 4 & 4 & 4 & 4 & 4 \\
$I\left(\nu_{6}\right)$ & 6 & 1 & 6 & 6 & 6 \\
\hline \hline
\end{tabular}

$$
A_{0}^{(\infty, 3)}=A_{e}^{(\infty)}-\Delta A^{(3)},
$$

and by analogy for $B$ and $C$. For comparison with the experimental results $B_{\text {eff }}$ reported in Table I, we introduce the average value $\bar{B}_{\text {eff }}^{(\infty, 3)}$,

$$
\bar{B}_{\text {eff }}^{(\infty, 3)}=\left[B_{0}^{(\infty, 3)}+C_{0}^{(\infty, 3)}\right] / 2,
$$

and the deviation $\Delta B_{\text {eff }}$,

$$
\Delta\left(B_{\text {eff }}\right)=\bar{B}_{\text {eff }}^{(\infty, 3)}-B_{\text {eff }}
$$

of the theoretical results $\bar{B}_{\text {eff }}^{(\infty, 3)}$ from the experimental counterparts $B_{\text {eff }}$. Table I shows that the $a b$ initio rotational constants agree very well with the experimentally determined values, exhibiting $\Delta\left(B_{\text {eff }}\right)$ of $12-14 \mathrm{MHz}$ (approximately $0.1 \%$ ) for the HONC isotopologues.

In Table II, we summarize the harmonic $\omega_{i}$ frequencies, the fundamental $\nu_{i}$ frequencies computed from the anharmonic force field by second order perturbation theory, and the infrared intensities derived within the double harmonic approximation. Table III provides the centrifugal distortion constants in Watson's S reduction. These two tables furnish additional information, potentially useful for future highresolution rotational spectroscopic studies on vibrationally excited HONC.

In accord with Table II, the most intense infrared transitions are to be expected in the region of the stretching $\nu_{1}(\mathrm{H}-\mathrm{O})$ frequency, followed by the $\nu_{3}(\mathrm{H}-\mathrm{O}-\mathrm{N}$ bend $)$ transition of an almost halved intensity. The $\left(\nu_{5}, \nu_{6}\right)$ wave number pair for the heavy-atom bending $\nu_{5}$ mode and the torsional $\nu_{6}$ mode calculated to be $\left(321 \mathrm{~cm}^{-1}, 273 \mathrm{~cm}^{-1}\right)$ for HONC is smaller than both $\left(576 \mathrm{~cm}^{-1}, 650 \mathrm{~cm}^{-1}\right)$ for HNCO and $\left(448 \mathrm{~cm}^{-1}, 504 \mathrm{~cm}^{-1}\right)$ for HOCN. ${ }^{40}$ Although the $\nu_{5}$ and $\nu_{6}$ modes of HONC possess a very low infrared spectral intensity in Table II, they are important in both the overall internal dynamics of real vibrating and rotating HONC (due to angular momentum conservation) and in the kinetic behavior (due to their major contribution to the number and density of states).

The vibrational $\nu_{1}$ and $\nu_{3}$ modes involve hydrogen and are sensitive to deuteration, see Table II. The intensity ratio $R_{\nu_{1}}=I_{\mathrm{DO}} / I_{\mathrm{HO}}$ of 0.50 for $\nu_{1}$ readily indicates the highly localized nature of the $\mathrm{OH}(\mathrm{D})$ vibration. This can be demonstrated as follows. Making use of the normal coordinate $Q_{\mathrm{XY}}$ of the $\mathrm{X}-\mathrm{Y}$ vibration written as $\sqrt{\mathcal{M}_{\mathrm{XY}}} r(\mathrm{X}-\mathrm{Y})$ in the local mode picture, where $\mathcal{M}_{\mathrm{XY}}$ is the reduced mass and $r(\mathrm{X}-\mathrm{Y})$ the internal coordinate, we may rewrite the intensity ratio

TABLE III. Centrifugal distortion constants (in megahertz) of Watson's S-reduced Hamiltonian, obtained from the $\operatorname{CCSD}(\mathrm{T}) / \mathrm{cc}-\mathrm{pCVTZ}$ calculations by the use of perturbation theory.

\begin{tabular}{lccccc}
\hline \hline Property & $\mathrm{HONC}$ & $\mathrm{DONC}$ & $\mathrm{HON}{ }^{13} \mathrm{C}$ & $\mathrm{HO}^{15} \mathrm{NC}$ & $\mathrm{H}^{18} \mathrm{ONC}$ \\
\hline $10^{3} D_{J}$ & 5.79 & 4.90 & 5.30 & 5.79 & 5.34 \\
$D_{K}$ & 325 & 43.2 & 332 & 355 & 356 \\
$D_{J K}$ & 0.604 & 0.682 & 0.555 & 0.582 & 0.482 \\
$10^{4} d_{1}$ & -1.11 & -1.39 & -0.99 & -1.13 & -1.02 \\
$10^{4} d_{2}$ & -0.15 & -0.39 & -0.13 & -0.15 & -0.12 \\
\hline \hline
\end{tabular}




$$
R_{\nu_{1}}=\left|\left(d \mu / d Q_{\mathrm{DO}}\right)_{e}\right|^{2} /\left|\left(d \mu / d Q_{\mathrm{HO}}\right)_{e}\right|^{2}
$$

in the local mode limit as

$$
{ }^{l m} R_{\nu_{1}}=\mathcal{M}_{\mathrm{HO}} / \mathcal{M}_{\mathrm{DO}}=m_{\mathrm{H}} m_{\mathrm{DO}} / m_{\mathrm{D}} m_{\mathrm{HO}}=0.53,
$$

where $m_{\mathrm{X}}$ and $m_{\mathrm{XY}}$ denote the mass of $\mathrm{X}$ and $\mathrm{XY}$, respectively. The theoretical $R_{\nu_{1}}$ value of 0.50 for HONC closely resembles the expected local mode result ${ }^{l m} R_{\nu_{1}}$. Based on similar arguments and the bond-distance-bond-angle expression of the reduced mass for the bending vibration, ${ }^{41}$ we obtain $R_{\nu_{3}}=0.63$ and ${ }^{l m} R_{\nu_{3}}=0.57$ for $\nu_{3}$, indicating a predominant local mode nature also for this bending mode. Inclusion of anharmonic contributions of the potential energy, in particular coupling with $\nu_{2}$ and $\nu_{1}$, may lead to a less localized character of $\nu_{3}$; this can influence spectral intensities in the case of strong anharmonic resonances.

Pinnavaia et al. ${ }^{4}$ suggested for the main isotopologue HONC a Fermi resonance of $\nu_{2}$ with $2 \nu_{4}$, a Coriolis resonance between $\nu_{5}$ and $\nu_{6}$, and a Darling-Dennison resonance of $\nu_{5}$ with $\nu_{6}$. In contrast, our ab initio calculations for the five HONC isotopologues leave room for a Fermi resonance of $\nu_{2}$ with $2 \nu_{3}$ only for DONC.

The centrifugal distortion constant $D_{J}$ of $5.8 \mathrm{kHz}$ for HONC in Table III is somewhat larger than $D_{J}=3.4 \mathrm{kHz}$ for $\mathrm{HNCO}$ and $D_{J}=4.1 \mathrm{kHz}$ for HOCN, obtained at the same $\operatorname{CCSD}(\mathrm{T}) /$ cc-pCVTZ level of theory. ${ }^{40}$ The constant $D_{K}$ of $325 \mathrm{MHz}$ for $\mathrm{HONC}$ is larger than $D_{K}=254 \mathrm{MHz}$ for $\mathrm{HOCN},{ }^{40}$ but much smaller than the experimentally derived value $D_{K}=5940 \mathrm{MHz}$ for $\mathrm{HNCO}$, known to be quasilinear. ${ }^{42}$ The value of $D_{K}$ is primarily defined by the quartic centrifugal distortion constant $\tau_{\text {aaaa }}$, found to be -1303 and $-176 \mathrm{MHz}$ for, respectively, HONC and DONC, such that a decrease in $D_{K}$ by a factor of 7.5 upon deuteration appears quite reasonable.

\section{Nuclear quadrupole coupling constants}

The nuclear quadrupole coupling constants $\chi_{i j}$ for the ${ }^{14} \mathrm{~N}(\mathrm{I}=1)$ and $\mathrm{D}(\mathrm{I}=1)$ nuclei are computed at the $\mathrm{CCSD}(\mathrm{T}) /$ cc-pCV5Z level of theory for the best $\operatorname{CCSD}(\mathrm{T})$ estimate structure of Fig. 2. These results are collected in Table IV. The theoretical results for $\chi_{i j}$ are not vibrationally averaged. Nuclear quadrupole coupling constants computed for HONC at the optimum $\operatorname{CCSD}(\mathrm{T}) / \mathrm{cc}$-pCV5Z geometry were already reported in Table 2 of Ref. 7. The latter structure and the best CCSD(T) estimate structure of Fig. 2 agree within $0.0003 \AA$ and $0.06^{\circ},{ }^{30}$ yielding nuclear quadrupole constants that agree within $1 \mathrm{kHz}$.

At the site of a specific nucleus $A$, the electric field gradient tensor $q$ in the principal $a, b, c$ axis system was computed first. The multiplication of the $q_{i j}$ components by the nuclear quadrupole moment $Q(A)$ of the nucleus A provides the quadrupole coupling tensor $\chi$,

$$
\chi_{i j}(\mathrm{~A})=[e Q(\mathrm{~A}) / h] q_{i j}(\mathrm{~A}),
$$

at the site of A for $i, j=a, b, c$, where $e$ and $h$ denote the elementary charge and Planck's constant, respectively. The tensors $\chi$ and $q$ are symmetric and traceless, such that e.g., $\chi_{i i}+\chi_{j j}+\chi_{k k}=0$ holds. From Eq. (6), we obtain
TABLE IV. Nuclear quadrupole coupling constants $\chi_{\alpha \beta}\left({ }^{14} \mathrm{~N}\right)$ and $\chi_{\alpha \beta}(\mathrm{D})$ (in megahertz) obtained experimentally (superscript exp) and computed from the correlated $\operatorname{CCSD}(\mathrm{T}) / \mathrm{cc}-\mathrm{pCV} 5 \mathrm{Z}$ density matrices at the best estimate structure displayed in Fig. 2. The numbers in parentheses given along with the experimental values are the estimated uncertainties $(1 \sigma)$ in units of the

\begin{tabular}{|c|c|c|c|c|}
\hline Property & HONC & DONC & $\mathrm{HON}^{13} \mathrm{C}$ & $\mathrm{H}^{18} \mathrm{ONC}$ \\
\hline${ }^{\exp } \boldsymbol{\chi}_{\boldsymbol{a} a \boldsymbol{a}}\left({ }^{14} \mathbf{N}\right)$ & $3.267(4)$ & $3.238(5)$ & $3.268(4)$ & $3.271(4)$ \\
\hline$\chi_{a a}\left({ }^{14} \mathrm{~N}\right)$ & 3.224 & 3.196 & 3.224 & 3.227 \\
\hline$\Delta \chi_{a a}$ & & 0.028 & 0.0 & -0.003 \\
\hline$\chi_{b b}\left({ }^{14} \mathrm{~N}\right)$ & -1.854 & -1.826 & -1.853 & -1.857 \\
\hline$\chi_{a b}\left({ }^{14} \mathrm{~N}\right)$ & -0.474 & -0.604 & -0.476 & -0.457 \\
\hline$\chi_{c c}\left({ }^{14} \mathrm{~N}\right)$ & -1.370 & -1.370 & -1.370 & -1.370 \\
\hline$\delta_{i s o}$ & & 1.5 & 0.0 & 0.2 \\
\hline$\theta(\alpha, a)$ & 174.7 & 173.2 & 174.7 & 174.9 \\
\hline$\theta\left(\alpha, \mathbf{r}_{\mathrm{ON}}\right)$ & 0.4 & 0.4 & 0.4 & 0.4 \\
\hline${ }^{\exp } \boldsymbol{\chi}_{\boldsymbol{a} a}(\mathbf{D})$ & & $-0.063(8)$ & & \\
\hline$\chi_{a a}(\mathrm{D})$ & & -0.056 & & \\
\hline$\chi_{b b}(\mathrm{D})$ & & 0.229 & & \\
\hline$\chi_{a b}(\mathrm{D})$ & & 0.142 & & \\
\hline$\chi_{c c}(\mathrm{D})$ & & -0.172 & & \\
\hline$\theta(\alpha, a)$ & & 67.6 & & \\
\hline$\theta\left(\alpha, \mathbf{r}_{\mathrm{DO}}\right)$ & & 177.4 & & \\
\hline
\end{tabular}
last significant digit. For the definition of $\Delta \chi_{a a}$ and the angles $\delta_{i s o}$ and $\theta$, see the main text (Sec. II C).

$$
\chi_{i j}\left({ }^{14} \mathrm{~N}\right)[\mathrm{MHz}]=4.80268 q_{i j}\left({ }^{14} \mathrm{~N}\right)\left[E_{h} / a_{0}^{2}\right]
$$

and

$$
\chi_{i j}(\mathrm{D})[\mathrm{MHz}]=0.67200 q_{i j}(\mathrm{D})\left[E_{h} / a_{0}^{2}\right]
$$

using $Q\left({ }^{14} N\right)=2.044 \mathrm{fm}^{2}$ and $Q(D)=0.286 \mathrm{fm}^{2}$, both taken from a "year-2008" compilation of the known spectroscopic nuclear quadrupole moments by Pyykkö. ${ }^{43}$

The diagonalization of the nuclear quadrupole coupling tensor yields the eigenvalues $\chi_{\alpha \alpha}, \chi_{\beta \beta}, \chi_{\gamma \gamma}$, defined such that $\left|\chi_{\alpha \alpha}\right| \geq\left|\chi_{\beta \beta}\right| \geq\left|\chi_{\gamma \gamma}\right|$, which provide the asymmetry parameter $\eta$ introduced as

$$
\eta=\left(\chi_{\gamma \gamma}-\chi_{\beta \beta}\right) / \chi_{\alpha \alpha} \text {. }
$$

The latter parameter quantifies the deviation from a cylindrical charge distribution about a specific nucleus. At the site of nitrogen, the eigenvalues of the tensor $\chi$ are calculated to be $\chi_{\alpha \alpha}\left({ }^{14} \mathrm{~N}\right)=3.268 \mathrm{MHz}, \quad \chi_{\beta \beta}\left({ }^{14} \mathrm{~N}\right)=-1.898 \mathrm{MHz}, \quad$ and $\chi_{\gamma \gamma}\left({ }^{14} \mathrm{~N}\right)=-1.370 \mathrm{MHz}$, giving thus $\eta\left({ }^{14} \mathrm{~N}\right)$ of 0.16 . For deuterium, we obtain eigenvalues of $\chi_{\alpha \alpha}(\mathrm{D})$ $=0.287 \mathrm{MHz}, \quad \chi_{\beta \beta}(\mathrm{D})=-0.172 \mathrm{MHz}, \quad$ and $\quad \chi_{\gamma \gamma}(\mathrm{D})=$ $-0.115 \mathrm{MHz}$, such that $\eta(\mathrm{D})=0.20$. Nonzero values of $\eta$ are characteristic of nonlinear bonds.

In Table IV, we also provide the angle $\theta(\alpha, a)$ between the inertial $a$ axis and the principal quadrupole $\alpha$ axis, as well as the angle $\delta_{i s o}$ between the $a$ axis of HONC and the $a$ axis of its isotopologues. The angles $\theta\left(\alpha, \mathbf{r}_{\mathrm{ON}}\right)$ and $\theta\left(\alpha, \mathbf{r}_{\mathrm{HO}}\right)$ of the principal quadrupole $\alpha$ axis with the bond distance vectors $\mathbf{r}_{\mathrm{ON}}=\mathbf{r}_{\mathrm{O}}-\mathbf{r}_{\mathrm{N}}$ and $\mathbf{r}_{\mathrm{DO}}=\mathbf{r}_{\mathrm{D}}-\mathbf{r}_{\mathrm{O}}$ are listed for the ${ }^{14} \mathrm{~N}$ and $\mathrm{D}$ case, respectively. As seen in the table, the principal $\alpha$ axis almost coincides with $\mathbf{r}_{\mathrm{ON}}$ in the case of ${ }^{14} \mathrm{~N}$ and with $\mathbf{r}_{\mathrm{DO}}$ in the case of deuterium.

In Table IV, the theoretical values for $\chi_{a a}$ agree with the experimental results ${ }^{\text {exp }} \chi_{a a}$ within $42-44 \mathrm{kHz}$ for the ${ }^{14} \mathrm{~N}$ 


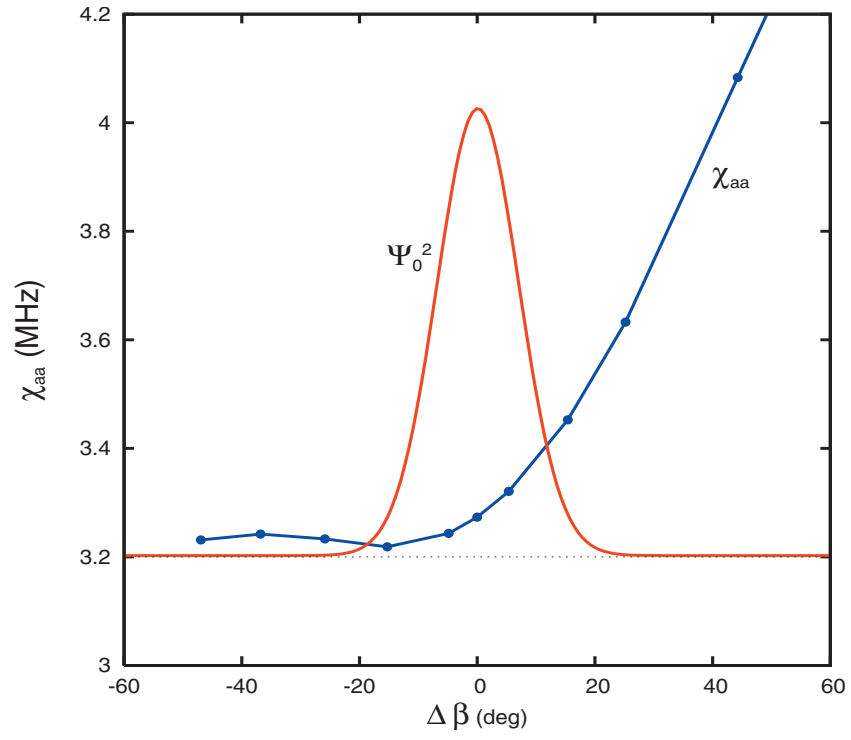

FIG. 3. Variation of the nitrogen nuclear quadrupole coupling constant $\chi_{a a}\left({ }^{14} \mathrm{~N}\right)$ of $\mathrm{HONC}$ along the minimum energy path in the direction of the bending angle $\beta$ involving hydrogen. Here, $\Delta \beta$ stands for the displacement $\beta-\beta_{e}$ from the equilibrium value $\beta_{e}$. The ground-state wave function probability amplitude $\Psi_{0}^{2}$ obtained from the corresponding one-dimensional calculation is additionally displayed.

nucleus and within $7 \mathrm{kHz}$ for deuterium. One may additionally note that the experimental and theoretical findings show the same trend with respect to the main species, $\mathrm{H}^{16} \mathrm{O}^{14} \mathrm{~N}^{12} \mathrm{C}$, as seen with the help of

$$
\Delta \chi_{a a}=\chi_{a a}(\mathrm{HONC})-\chi_{a a}(\text { isotope }) \text {. }
$$

The theoretical $\Delta \chi_{a a}$ values of 28,0 , and $-3 \mathrm{kHz}$ for nitrogen are in excellent agreement with the experimental $\Delta \chi_{a a}$ results of $29,-1$, and $-4 \mathrm{kHz}$ obtained for, respectively, DONC, $\mathrm{HON}^{13} \mathrm{C}$, and $\mathrm{H}^{18} \mathrm{ONC}$.

The magnitude of the nitrogen nuclear quadrupole coupling constant $\left|\chi_{a a}\left({ }^{14} \mathrm{~N}\right)\right|$ for HONC is larger than the respective values of 2.114, 2.872, and $0.245 \mathrm{MHz}$ found for HNCO, HOCN, and HCNO, respectively. ${ }^{7}$ The principal quadrupole component $\chi_{c c}\left({ }^{14} \mathrm{~N}\right)$ is negative for HONC; to be compared with $-1.559,0.795$, and $-0.147 \mathrm{MHz}$ for $\mathrm{HNCO}$, HOCN, and HCNO, respectively. ${ }^{7}$

The nuclear quadrupole constants of HONC are now compared with the corresponding values for HNC. A recent theoretical $\operatorname{CCSD}(\mathrm{T}) / \mathrm{cc}-\mathrm{pCVTZ}$ result for the equilibrium $\chi_{a a}\left({ }^{14} \mathrm{~N}\right)$ of $\mathrm{HNC}$ is given as $0.353 \mathrm{MHz} .{ }^{44}$ Our $\operatorname{CCSD}(\mathrm{T}) /$ aug-cc-pCVQZ value for this molecule amounts, however, to $\chi_{a a}\left({ }^{14} \mathrm{~N}\right)=0.317 \mathrm{MHz}$, whereas we obtain $\chi_{a a}(\mathrm{D})$ of $0.279 \mathrm{MHz}$. In any case, we see that the equilibrium nuclear quadrupole constants for HONC appear to be one order of magnitude larger than those of $\mathrm{HNC}$, exhibiting also a different sign in the case of $\chi_{a a}(\mathrm{D})$. For the ground vibrational state of HNC, Wong ${ }^{44}$ computed $\chi_{a a}\left({ }^{14} \mathrm{~N}\right)$ of 0.296 $\mathrm{MHz}$, which is thus by approximately $60 \mathrm{kHz}(16 \%)$ smaller than his equilibrium result due to vibrational averaging. The experimentally derived $\chi_{a a}\left({ }^{14} \mathrm{~N}\right)$ and $\chi_{a a}(\mathrm{D})$ values for the ground vibrational state of HNC are reported to be $0.264 \mathrm{MHz}$ and $0.262 \mathrm{MHz}$, respectively. ${ }^{45}$

In the case of nonlinear HONC, vibrational averaging may be expected to play a somewhat less important role since HONC possesses a pronounced barrier to linearity of ca. $16700 \mathrm{~cm}^{-1}$, as seen in Fig. 3 of Ref. 30, which shows the $\mathrm{CCSD}(\mathrm{T}) / \mathrm{cc}-\mathrm{pVQZ}$ minimum energy path (MEP) along the bond angle HON of HONC. The variation of $\chi_{a a}\left({ }^{14} \mathrm{~N}\right)$ along this MEP depicted in Fig. 3 shows a steep increase of more than $1 \mathrm{MHz}$ in $\chi_{a a}\left({ }^{14} \mathrm{~N}\right)$ upon straightening of the bending angle involving hydrogen. One-dimensional calculations carried out for $\beta$ in the framework of the orthogonal diatom-diatom description of the internal molecular geometry ${ }^{46}$ give an estimate of $+15 \mathrm{kHz}(0.5 \%)$ for the ground-state vibrational correction to $\chi_{a a}\left({ }^{14} \mathrm{~N}\right)$ due to this bending mode. The heavy-atom bending vibration does not contribute much to the vibrational averaging, since $\chi_{a a}\left({ }^{14} \mathrm{~N}\right)$ exhibits only a negligible variation of several kilohertz along the corresponding minimum energy path. More details on these calculations will be published elsewhere. ${ }^{40}$

\section{Electron density}

The nuclear quadrupole coupling constant is the spectroscopic measure of the interaction between the nuclear quadrupole moment and the molecular electric field gradient at the specific nuclear site. To qualitatively understand this effect, as well as a possible binding mechanism, several results regarding the electron subspace of $\mathrm{HONC}$ are presented here. For more details on this topic, see Ref. 7.

The individual basis function (orbital) populations are given in Table $\mathrm{V}$, where $p_{z}$ and $p_{y}$ are the in-plane $p$ orbitals, whereas $p_{x}$ stands for the out-of-plane functions. The table clearly shows a decrease in the $p_{z}$ and $s$ characters and an increase in the $p_{x}$ and $p_{y}$ characters for both nitrogen and oxygen with respect to the free atom situation. This is an indication of a hybridization involving $p$ orbitals ${ }^{47}$ ( $\pi$ type binding). Although the orbital population at the carbon center is almost unchanged with respect to free $\mathrm{C}$, a small increase of 0.08 electrons for $p_{z}$ of $\mathrm{C}$ indicates some $s p$ hybridization.

The nuclear quadrupole interaction results from the nonspherical charge distribution over hybrid orbitals, ${ }^{47,48}$ the spherically symmetric closed-shell $s$ electrons contributing nothing. ${ }^{48}$ In accord with Fig. 4 showing the total electron distribution and Table $\mathrm{V}$, a nearly cylindrically symmetric electron distribution in the valence shell of nitrogen and

TABLE V. Population analysis of the total CCSD(T)/cc-pCV5Z electron density by the basis function type.

\begin{tabular}{lcccccc}
\hline \hline Center & $s$ & $p_{z}$ & $p_{y}$ & $p_{x}$ & Total & Charge \\
\hline $\mathrm{H}$ & 0.59 & 0.02 & 0.02 & 0.05 & 0.69 & +0.31 \\
$\mathrm{O}$ & 3.79 & 1.08 & 1.45 & 1.83 & 8.24 & -0.24 \\
$\mathrm{~N}$ & 3.25 & 0.82 & 1.35 & 1.31 & 6.86 & +0.14 \\
$\mathrm{C}$ & 4.04 & 0.74 & 0.61 & 0.68 & 6.21 & -0.21 \\
\hline \hline
\end{tabular}




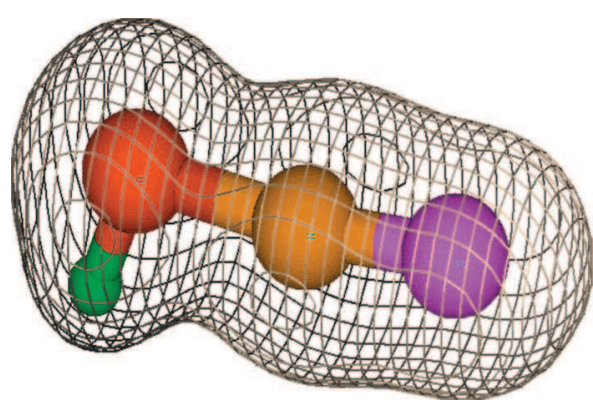

FIG. 4. Total electron density of HONC. The contour surface of magnitude 0.025 a.u. is shown.

largely asymmetric electron distribution in the valence shell of oxygen may readily be expected for $\mathrm{HONC}$, resulting in an electric field gradient component $q_{a a}\left({ }^{14} \mathrm{~N}\right)$ of $0.671 E_{h} / a_{0}^{2}$ markedly smaller than $q_{a a}\left({ }^{17} \mathrm{O}\right)$ of $2.417 E_{h} / a_{0}^{2}$. Since the magnitude of the nuclear quadrupole moment $Q\left({ }^{17} \mathrm{O}\right)$ of $-2.558 \mathrm{fm}^{2}$ (Ref. 43) is comparable to $Q\left({ }^{14} \mathrm{~N}\right.$ ), it follows $\left|\chi_{a a}\left({ }^{14} \mathrm{~N}\right)\right|<\left|\chi_{a a}\left({ }^{17} \mathrm{O}\right)\right|$, as verified by our calculations reported elsewhere, ${ }^{7}$ where we find $\chi_{a a}\left({ }^{17} \mathrm{O}\right)$ of $-14.51 \mathrm{MHz}$.

The highest occupied bonding molecular $9 a^{\prime}$ orbital of HONC is compared with the highest occupied molecular $5 \sigma^{+}$ orbital of HNC in Fig. 5. The molecular $9 a^{\prime}$ orbital of HONC at $-0.491 E_{h}$ lies $0.023 E_{h}$ below the highest occupied nonbonding out-of-plane $2 a^{\prime \prime}$ orbital at $-0.468 E_{h}$ shown in Fig. 1. The molecular orbitals of Fig. 5 are very similar, both clearly exhibiting a promotion of $p_{z}$ electrons from nitrogen toward carbon and an electron cloud on carbon. In consequence, HONC and HNC possess similar equilibrium $r_{e}(\mathrm{CN})$ bond lengths [1.173 $\AA$ versus $1.169 \AA$ (Ref. $34)]$ due to a similar type of binding, schematically described as $\mathrm{N}^{+} \equiv \mathrm{C}^{-}$.

Figure 5, as well as Figs. 1 and 4, were drawn with the help of the pre- and postprocessing program MOLDEN. ${ }^{49}$

\section{LABORATORY MEASUREMENTS AND RESULTS}

The rotational spectrum of HONC was detected with the same Fourier transform microwave spectrometer ${ }^{50}$ used to detect cyanic acid, HOCN, and many other reactive molecules of chemical and astronomical interest. Many of these

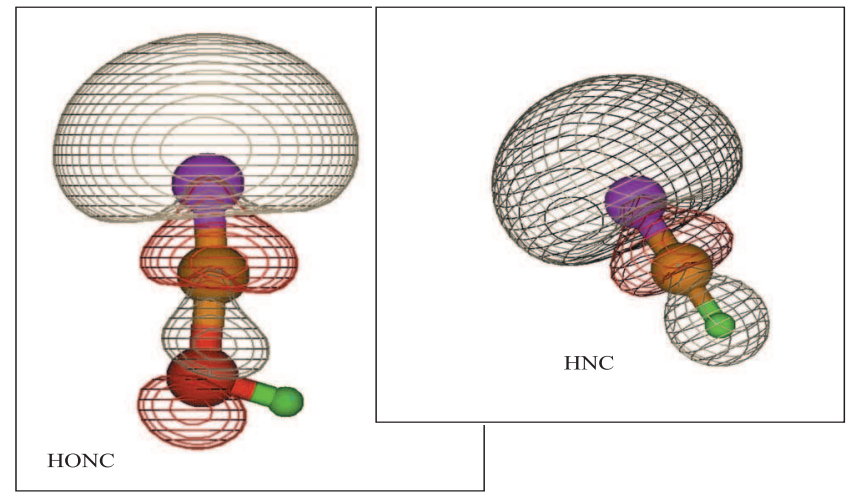

FIG. 5. Highest occupied bonding molecular $9 a^{\prime}$ orbital of HONC (left) and highest occupied molecular $5 \sigma^{+}$orbital of HNC (right). The contour surfaces of magnitude 0.025 and -0.025 are shown.

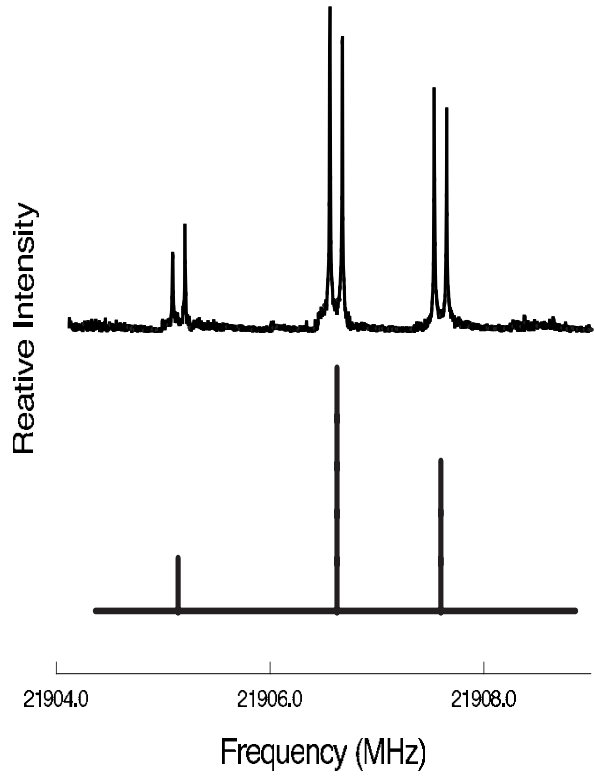

FIG. 6. Observed (top) and calculated (bottom) spectrum of the fundamental rotational transition of HONC, showing well resolved nitrogen hyperfine structure. The observed spectrum is a synthesis of several settings of the Fabry-Pérot cavity which, owing to the high- $Q$ of the cavity at this frequency $(Q \sim 10000)$, differ in frequency by $0.3 \mathrm{MHz}$. Each hyperfine component has a double-peaked line shape, the result of the Doppler shift of the Mach 2 molecular beam relative to the two traveling waves that compose the confocal mode of the Fabry-Pérot. The total integration time was 5 min. Shown beneath the observed spectrum is a calculated stick diagram derived from the best-fit constants in Tables I and IV.

are radicals and carbenes, but positively and negatively charged species have been studied as well. Reactive molecules are created in the throat of a small supersonic nozzle by applying a low-current dc discharge to a short gas pulse created by a fast mechanical valve.

Like fulminic and cyanic acid, isofulminic acid is a closed-shell asymmetric rotor near the prolate limit $(\kappa=-0.9994)$, with a nearly linear heavy-atom backbone closely parallel to the $a$ inertial axis (see Fig. 2), and a large rotational constant $A$ (see Table I). Both intrastack $a$-type $\left(\Delta K_{a}=0\right)$ and interstack $b$-type $\left(\Delta K_{a}=\Delta K_{c}= \pm 1\right)$ rotational transitions are allowed, and the calculated dipole moments along the two principal axes are fairly large $\left(\mu_{a}=3.13 \mathrm{D}\right.$, $\mu_{b}=-1.54 \mathrm{D}$; see Fig. 2). At the very low rotational temperature of our molecular beam $\left(T_{\text {rot }} \sim 3 \mathrm{~K}\right)$, however, only levels in the $K_{a}=0$ ladder will be thermally populated, and the rotational spectrum of HONC simplifies to that of a linear molecule, with lines separated in frequency by approximately $(B+C) \sim 22 \mathrm{GHz}$. Because of the relatively low frequency ceiling of our FTM spectrometer $(<42 \mathrm{GHz})$, only the fundamental rotational transition $\left(1_{0,1} \rightarrow 0_{0,0}\right)$ of HONC and its isomers is accessible with our present instrumentation. At the high spectral resolution (of order $0.1 \mathrm{ppm}$ ) which is readily achieved in our molecular beam experiments, however, the fundamental rotational line of HONC should possess a readily identifiable spectral fingerprint: a closely spaced, but well-resolved triplet with a characteristic 1:5:3 intensity ratio between the three lines, owing to nitrogen quadrupole hyperfine structure.

Our laboratory search for HONC was guided by rota- 
TABLE VI. Measured hyperfine structure in the fundamental rotational transition of $\mathrm{HONC}, \mathrm{H}^{18} \mathrm{ONC}$, and $\mathrm{HON}^{13} \mathrm{C}$ (in megahertz).

\begin{tabular}{cccc}
\hline \hline$F^{\prime} \rightarrow F^{\mathrm{a}}$ & $\mathrm{HONC}$ & $\mathrm{HON}^{13} \mathrm{C}$ & $\mathrm{H}^{18} \mathrm{ONC}$ \\
\hline $1 \rightarrow 1$ & 21905.146 & 21054.962 & 20814.337 \\
$2 \rightarrow 1$ & 21906.620 & 21056.435 & 20815.813 \\
$0 \rightarrow 1$ & 21907.596 & 21057.413 & 20816.790 \\
\hline \hline
\end{tabular}

${ }^{\mathrm{a}}$ Note-Estimated measurement uncertainties are $2 \mathrm{kHz}$.

tional constants derived from the high-level molecular structure calculations described in Sec. II B. Because zero-point corrections are generally small, the equilibrium $\left(B_{e}\right)$ and vibrationally corrected $\left(B_{0}\right)$ rotational constants differ by less than $0.4 \%$, which amounts to a search of at most $\pm 80 \mathrm{MHz}$ near 21.9 GHz. Before undertaking the search, the discharge conditions were optimized on the corresponding transition of $\mathrm{HNCO}$, which was produced in a $800 \mathrm{~V}$ dc discharge in the throat of the supersonic nozzle of the spectrometer through a mixture of water $(0.035 \%)$, and cyanogen $(\mathrm{NCCN}, 0.03 \%)$ heavily diluted in neon. Typical gas flow rates were $30 \mathrm{~cm}^{3} /$ min at standard temperature and pressure at a $6 \mathrm{~Hz}$ pulse repetition rate, with pulses $300 \mu$ s in duration, and a stagnation pressure of 2.5 ktorr behind the nozzle. Under these conditions, the fundamental rotational transition of HNCO $(22.0 \mathrm{GHz})$ is very intense, but the same line of HOCN $(21.0 \mathrm{GHz})$ and HCNO $(22.9 \mathrm{GHz})$ is also readily observed.

Only one unidentified triplet (see Fig. 6 and Table VI) was found in the search, and it almost certainly arises from HONC because (1) the measured frequency is very close to that calculated from two high-level $a b$ initio equilibrium structure calculations, lying $0.12 \%$ lower than that predicted in Table I, and $0.10 \%$ lower than that predicted by Schuurman et al. ${ }^{5}$ at a similarly high level of theory and (2) the measured quadrupole coupling constant $\chi_{a a}=3.267 \mathrm{MHz}$ is extremely close to that predicted (3.224 MHz; Table IV). Additional evidence of the identification is provided by the observation of the same transition of $\mathrm{H}^{18} \mathrm{ONC}, \mathrm{HO}^{13} \mathrm{NC}$, and

TABLE VII. Measured hyperfine structure in the fundamental rotational transition of DONC (in megahertz).

\begin{tabular}{ccc}
\hline \hline$F_{1}^{\prime} \rightarrow F_{1}$ & $F^{\prime} \rightarrow F$ & Frequency $^{\mathrm{a}}$ \\
\hline \multirow{2}{*}{$1 \rightarrow 1$} & $1 \rightarrow 0$ & 20822.026 \\
& $1 \rightarrow 1$ & 20822.026 \\
$1 \rightarrow 2$ & 20822.026 \\
$2 \rightarrow 1$ & $2 \rightarrow 1$ & 20823.477 \\
& $2 \rightarrow 2$ & 20823.477 \\
& $3 \rightarrow 2$ & 20823.490 \\
& $1 \rightarrow 0$ & 20823.498 \\
& $1 \rightarrow 1$ & 20823.498 \\
& $1 \rightarrow 2$ & 20823.498 \\
& $0 \rightarrow 1$ & 20824.441 \\
& $2 \rightarrow 1$ & 20824.454 \\
& $2 \rightarrow 2$ & 20824.454 \\
& $1 \rightarrow 0$ & 20824.464 \\
& $1 \rightarrow 1$ & 20824.464 \\
& $1 \rightarrow 2$ & 20824.464
\end{tabular}

${ }^{\mathrm{a}}$ Note—Estimated measurement uncertainties are $3 \mathrm{kHz}$.
DONC at the expected isotopic shifts with essentially the same $\chi_{a a}$ as that measured for the normal species. For DONC, additional quadrupole hyperfine structure from the deuterium was resolved (Table VII), and as Table IV shows the theoretical and experimental quadrupole coupling constants for deuterium and nitrogen are in excellent agreement. For the $\mathrm{H}^{18} \mathrm{ONC}$ isotopic measurements, ${ }^{18} \mathrm{O}_{2}$ was used as a precursor gas together with methyl cyanide $\left(\mathrm{CH}_{3} \mathrm{CN}\right)$; for HON ${ }^{13} \mathrm{C}$, carbon-13 methyl cyanide $\left(\mathrm{CH}_{3}{ }^{13} \mathrm{CN}\right)$ was used with water; and for DONC, heavy water $\left(\mathrm{D}_{2} \mathrm{O}\right)$ was used with cyanogen. A search for $\mathrm{HO}^{15} \mathrm{NC}$ was also undertaken using methanol and ${ }^{15} \mathrm{~N}_{2}$, and several lines were found near the predicted isotopic shift; owing to the lack of quadrupole moment for ${ }^{15} \mathrm{~N}$, it was not possible to unambiguously identify which line arises from this isotopic species.

\section{DISCUSSION}

The present work firmly establishes the existence of the elusive isofulminic acid, and will permit a comparative study of all four isocyanic isomers. Although the data set is limited for $\mathrm{HONC}$, with the isotopic data in hand, an experimental $r_{0}$ structure for this bent chain can be derived assuming a nearly linear heavy-atom $\mathrm{ONC}$ backbone $\angle(\mathrm{ONC})=172.6^{\circ}$ and $\angle(\mathrm{HON})=105^{\circ}$. On this basis we derive bond lengths of $r_{\mathrm{CN}}^{0}=1.177(3) \AA, r_{\mathrm{NO}}^{0}=1.333(3) \AA$, and $r_{\mathrm{OH}}^{0}=0.958(3) \AA$. Making use of the ground-state vibrational corrections $\Delta B^{(3)}$ and $\Delta C^{(3)}$ from Table I in combination with the experimental results $B_{\text {eff }}$, we obtain experimental equilibrium $r_{e}$ bond lengths given by $r_{\mathrm{CN}}^{e}=1.1744(4) \AA, r_{\mathrm{NO}}^{e}=1.3304(4) \AA$, and $r_{\mathrm{OH}}^{e}=0.9605(4) \AA$. The latter results are in good agreement (to better than $\pm 0.005 \AA$ ) with those predicted from theory, Fig. 2, providing thus a further evidence that the real "carboxime" $"$ with the structure $\mathrm{HO}-\mathrm{N} \equiv \mathrm{C}$ has indeed been found. Analytical expressions for the principal moments of inertia in the bond-distance-bond-angle description and for their partial derivatives with respect to the geometrical parameters were employed here in combination with a Levenberg-Marquardt nonlinear least-squares algorithm ${ }^{52}$ to compute the experimental equilibrium bond lengths $r_{\mathrm{CN}}^{e}, r_{\mathrm{NO}}^{e}$, and $r_{\mathrm{OH}}^{e}$.

It is rather remarkable that isofulminic acid, calculated to lie $84 \mathrm{kcal} / \mathrm{mol}$ or nearly $4 \mathrm{eV}$ above isocyanic acid, is readily produced in electric discharges starting from a wide variety of precursor gases containing $\mathrm{H}, \mathrm{C}, \mathrm{N}$, and $\mathrm{O}$. We estimate from line intensities that abundances relative to $\mathrm{HNCO}$ are $12 \%$ for $\mathrm{HOCN}, 2 \%$ for $\mathrm{HCNO}$, and $1 \%$ for HONC in the molecular beam. Now that all four isocyanic isomers have been detected spectroscopically, it may be worthwhile to systematically measure relative abundances with different precursor combinations. Such a study might clarify the production routes that are operative in our discharge source.

Now that a simple but fairly efficient method for in situ synthesis of HONC has been demonstrated here, it should be possible to extend present work to the millimeter and submillimeter wavelengths to determine individual rotational and centrifugal distortion constants. Because HONC possesses a sizable dipole moment along the $b$-axis 
$\left(\mu_{b}=-1.54 \mathrm{D}\right)$, a precise determination of the $A$ rotational constant should be possible. The IR vibrational spectrum of HONC is also of interest to test theoretical models of the potential energy surface and to determine the extent of quasilinearity for this highly energetic isomer. The calculated fundamental vibrational frequencies, infrared intensities, and centrifugal distortion constants in Tables II and III should serve as a useful guide.

Ground state rotational constants derived from coupled cluster calculations here and elsewhere agree to $\sim 0.1 \%$ or better with those measured for HONC and its three isomers, and a nearly comparable level of accuracy was recently achieved for another set of closed-shell molecules, the carbon-chain anions $\mathrm{C}_{2 n} \mathrm{H}^{-} \quad(n=1-4),{ }^{53-55} \mathrm{CN}^{-},{ }^{56}$ and $\mathrm{C}_{3} \mathrm{~N}^{-57}$. Such close agreement suggests that the same theoretical methods might be applied to predict accurate spectroscopic constants for many other closed-shell molecules. It remains to be seen if $0.1 \%$ is the limit of present theory or not, and to critically assess if theoretical methods can be refined even further, perhaps by a factor of 10 or more, i.e., $0.01 \%$. Even at the present level of accuracy, theoretical spectroscopic constants are an extremely helpful aid in the assignment of rotational spectra: they significantly reduce the frequency range of a laboratory search and well predict hyperfine structure.

Because HOCN and HCNO have recently been identified in space, detection of HONC is not out of the realm of possibility. It is calculated to lie only $14 \mathrm{kcal} / \mathrm{mol}$ higher in energy than $\mathrm{HCNO}$ and is comparably polar, so it may be found in warm regions where $\mathrm{HCNO}$ is abundant. If detected in space, the abundance ratios between HONC and other isomers may provide further insight into the complex gas and surface chemistry, which is thought to produce these isomers. The discovery of HCNO in space, for example, led Marcelino et al. ${ }^{17}$ to suggest that $\mathrm{CH}_{2}+\mathrm{NO} \rightarrow \mathrm{HCNO}+\mathrm{H}$ is a key reaction in the formation of fulminic acid, allowing them to reproduce the observed abundances of $\mathrm{HCNO}$ in both dark clouds and the low-mass star forming core L1527.

\section{ACKNOWLEDGMENTS}

M.M. and M.L. kindly acknowledge the access to the high performance computers of the Institut du Développement et des Ressources en Informatique Scientifique (IDRIS) through Grant No. 82170. The work in Cambridge is supported by NSF grant (Grant No. CHE-0701204) and NASA grant (Grant No. NNX08AE05G).

${ }^{1}$ J. H. Teles, G. Maier, B. A. Hess, Jr., L. J. Schaad, M. Winnewisser, and B. P. Winnewisser, Chem. Ber. 122, 753 (1989) (and references therein).

${ }^{2}$ A. D. McLean, G. H. Loew, and D. S. Berkowitz, J. Mol. Spectrosc. 64, 184 (1977).

${ }^{3}$ D. Poppinger, L. Radom, and J. A. Pople, J. Am. Chem. Soc. 99, 7806 (1977).

${ }^{4}$ N. Pinnavaia, M. J. Bramley, M.-D. Su, W. H. Green, and N. C. Handy, Mol. Phys. 78, 319 (1993).

${ }^{5}$ M. S. Schuurman, S. R. Muir, W. D. Allen, and H. F. Schaefer III, J. Chem. Phys. 120, 11586 (2004).

${ }^{6}$ M. Mladenović and M. Lewerenz, Chem. Phys. 343, 129 (2008).

${ }^{7}$ M. Mladenović, M. Elhiyani, and M. Lewerenz, J. Chem. Phys. 131, 034302 (2009).

${ }^{8}$ D. Buhl, L. E. Snyder, and J. Edrich, Nature (London) 243, 513 (1973).
${ }^{9}$ R. Kewley, K. V. L. N. Sastry, and M. Winnewisser, J. Mol. Spectrosc. 10, 418 (1963).

${ }^{10}$ J. M. Jackson, J. T. Armstrong, and A. H. Barrett, Astrophys. J. 280, 608 (1984).

${ }^{11}$ N. Q. Rieu, C. Henkel, J. M. Jackson, and R. Mauersberger, Astron. Astrophys. 241, L33 (1991).

${ }^{12}$ I. Zinchenko, C. Henkel, and R. Q. Mao, Astron. Astrophys. 361, 1079 (2000).

${ }^{13}$ M. Wang, C. Henkel, Y.-N. Chin, J. B. Whiteoak, M. H. Cunningham, R. Mauersberger, and D. Muders, Astron. Astrophys. 422, 883 (2004).

${ }^{14}$ S. Martín, M. A. Requena-Torres, J. Martín-Pintado, and R. Mauersberger, Astrophys. J. 678, 245 (2008).

${ }^{15}$ S. Brünken, C. A. Gottlieb, M. C. McCarthy, and P. Thaddeus, Astrophys. J. 697, 880 (2009).

${ }^{16}$ S. Brünken, A. Belloche, S. Martín, L. Verheyen, and K. M. Menten, "Interstellar HOCN in the Galactic center region," Astron. Astrophys. (submitted).

${ }^{17}$ N. Marcelino, N. J. Cernicharo, N. B. Tercero, and E. Roueff, Astrophys. J. 690, L27 (2009).

${ }^{18}$ B. P. Winnewisser, in Molecular Spectroscopy: Modern Research, edited by K. N. Rao (Academic, Orlando, 1985), Vol. 3, p. 321 (and references therein).

${ }^{19}$ M. Winnewisser, B. P. Winnewisser, I. R. Medvedev, F. C. De Lucia, S. C. Ross, and L. M. Bates, J. Mol. Struct. 798, 1 (2006) (and references therein).

${ }^{20}$ M. E. Jacox and D. E. Milligan, J. Chem. Phys. 40, 2457 (1964).

${ }^{21}$ V. E. Bondybey, J. H. English, C. W. Mathews, and R. J. Contolini, J. Mol. Spectrosc. 92, 431 (1982).

${ }^{22}$ G. Maier, J. H. Teles, B. A. Hess, Jr., and L. J. Schaad, Angew. Chem., Int. Ed. Engl. 27, 938 (1988).

${ }^{23}$ G. Maier, A. Bothur, J. Eckwert, H. P. Reisenauer, and T. Stumpf, Liebigs. Ann. 1997, 2505 (1997).

${ }^{24}$ C. Wentrup, B. Gerecht, and H. Briehl, Angew. Chem., Int. Ed. Engl. 18, 467 (1979).

${ }^{25}$ D. McNaughton and C. J. Evans, Z. Phys. Chem. 214, 1313 (2000).

${ }^{26}$ D. Poppinger and L. Radom, J. Am. Chem. Soc. 100, 3674 (1978).

${ }^{27}$ C. Wentrup, B. Gerecht, D. Laqua, H. Briehl, H.-W. Winter, H. P. Reisenauer, and M. Winnewisser, J. Org. Chem. 46, 1046 (1981).

${ }^{28}$ A. M. Mebel, A. Luna, M. C. Lin, and K. Morokuma, J. Chem. Phys. 105, 6439 (1996)

${ }^{29}$ Y.-Q. Ding, D.-C. Fang, and R.-Z. Liu, Mol. Phys. 103, 767 (2005).

${ }^{30}$ M. Mladenović, M. Elhiyani, and M. Lewerenz, J. Chem. Phys. 130, 154109 (2009).

${ }^{31}$ J. F. Stanton, J. Gauss, J. D. Watts, P. G. Szalay, R. J. Bartlett with contributions from A. A. Auer, D. B. Bernholdt, O. Christiansen, M. E. Harding, M. Heckert, O. Heun, C. Huber, D. Jonsson, J. Jusélius, W. J. Lauderdale, T. Metzroth, C. Michauk, D. P. O'Neill, D. R. Price, K. Ruud, F. Schiffmann, M. E. Varner, J. Vázquez and the integral packages MOLECULE (J. Almlöf and P. R. Taylor), PROPS (P. R. Taylor), and ABACUS (T. Helgaker, H. J. Aa. Jensen, P. Jørgensen, and J. Olsen). For the current version, see http://www.aces2.de.

${ }^{32}$ T. H. Dunning, Jr., J. Chem. Phys. 90, 1007 (1989).

${ }^{33}$ D. E. Woon and T. H. Dunning, Jr., J. Chem. Phys. 103, 4572 (1995).

${ }^{34}$ R. A. Creswell and A. G. Robiette, Mol. Phys. 36, 869 (1978).

${ }^{35} \mathrm{G}$. Herzberg, Infrared and Raman Spectra of Polyatomic Molecules, Molecular Spectra \& Molecular Structure, Vol. II, (corrected reprint of 1945 edition) (Krieger, Malabar FL, 1991).

${ }^{36}$ W. H. Hocking, M. C. L. Gerry, and G. Winnewisser, Can. J. Phys. 53, 1869 (1975).

${ }^{37}$ R. Takashi, K. Tanaka, and T. Tanaka, J. Mol. Spectrosc. 138, 450 (1989).

${ }^{38}$ G. L. Blackman, R. D. Brown, P. D. Godfrey, and H. I. Gunn, Nature (London) 261, 395 (1976)

${ }^{39}$ I. M. Mills, in Molecular Spectroscopy: Modern Research, edited by K. N. Rao and C. W. Mathews (Academic, New York, 1972), Vol. 3, p. 115.

${ }^{40}$ M. Mladenović, "Spectroscopic properties of the four most stable CHNO isomers from ab initio $\operatorname{CCSD}(\mathrm{T})$ studies," J. Chem. Phys. (in preparation).

${ }^{41}$ M. Mladenović, J. Chem. Phys. 112, 1082 (2000).

${ }^{42}$ A. V. Lapinov, G. Y. Golubiatnikov, V. N. Markov, and A. Guarnieri, Astron. Lett. 33, 121 (2007).

${ }^{43}$ P. Pyykkö, Mol. Phys. 106, 1965 (2008).

${ }^{44}$ B. M. Wong, Phys. Chem. Chem. Phys. 10, 5599 (2008).

${ }^{45}$ H. A. Bechtel, A. H. Steeves, and R. W. Field, Astrophys. J. 649, L53 
(2006).

${ }^{46}$ M. Mladenović, J. Chem. Phys. 112, 1070 (2000).

${ }^{47}$ B. P. Dailey, J. Phys. Chem. 57, 490 (1953).

${ }^{48}$ C. H. Townes and B. P. Dailey, J. Chem. Phys. 17, 782 (1949).

${ }^{49}$ G. Schaftenaar and J. H. Noordik, J. Comput.- Aided Mol. Des. 14, 123 (2000).

${ }^{50}$ M. C. McCarthy, W. Chen, M. J. Travers, and P. Thaddeus, Astrophys. J., Suppl. Ser. 129, 611 (2000) (and references therein).

${ }^{51}$ G. Maier, Pure Appl. Chem. 63, 275 (1991).

${ }^{52}$ W. H. Press, B. P. Flannery, S. A. Teukolsky, and W. T. Vetterling, $N u$ - merical Recipes (Cambridge University Press, Cambridge, 1986).

${ }^{53}$ M. C. McCarthy, C. A. Gottlieb, H. Gupta, and P. Thaddeus, Astrophys. J. 652, L141 (2006).

${ }^{54}$ H. Gupta, S. Brünken, F. Tamassia, C. A. Gottlieb, M. C. McCarthy, and P. Thaddeus, Astrophys. J. 655, L57 (2007)

${ }^{55}$ S. Brünken, C. A. Gottlieb, H. Gupta, M. C. McCarthy, and P. Thaddeus, Astron. Astrophys. 464, L33 (2007).

${ }^{56}$ C. A. Gottlieb, S. Brünken, M. C. McCarthy, and P. Thaddeus, J. Chem. Phys. 126, 191101 (2007)

${ }^{57}$ M. C. McCarthy and P. Thaddeus, J. Chem. Phys. 129, 054314 (2008). 